\title{
Optimal Algorithms for Crawling a Hidden Database in the Web.
}

\author{
Cheng Sheng ${ }^{1} \quad$ Nan Zhang $^{3} \quad$ Yufei Tao ${ }^{1,2} \quad$ Xin Jin $^{3}$ \\ ${ }^{1}$ Chinese University of Hong Kong ${ }^{2}$ Korea Advanced Institute of Science and Technology \\ \{csheng, taoyf\}@cse.cuhk.edu.hk taoyf@kaist.ac.kr \\ ${ }^{3}$ George Washington University \\ nzhang10@gwu.edu, xjin@gwmail.gwu.edu
}

\begin{abstract}
A hidden database refers to a dataset that an organization makes accessible on the web by allowing users to issue queries through a search interface. In other words, data acquisition from such a source is not by following static hyper-links. Instead, data are obtained by querying the interface, and reading the result page dynamically generated. This, with other facts such as the interface may answer a query only partially, has prevented hidden databases from being crawled effectively by existing search engines.

This paper remedies the problem by giving algorithms to extract all the tuples from a hidden database. Our algorithms are provably efficient, namely, they accomplish the task by performing only a small number of queries, even in the worst case. We also establish theoretical results indicating that these algorithms are asymptotically optimal - i.e., it is impossible to improve their efficiency by more than a constant factor. The derivation of our upper and lower bound results reveals significant insight into the characteristics of the underlying problem. Extensive experiments confirm the proposed techniques work very well on all the real datasets examined.
\end{abstract}

\section{INTRODUCTION}

It is known that existing search engines can reach only a small portion of the Internet. They crawl HTML pages interconnected with hyper-links, which constitute the so-called surface web. Nowadays, an increasing number of organizations (e.g., companies, governments, institutions, etc.) bring their data online, by allowing a public user to query their back-end databases through context-dependent web interfaces. More specifically, data acquisition is performed by interacting with the interface at runtime, as opposed to following hyper-links. As a result, those back-end databases cannot be effectively crawled by a search engine under

\begin{abstract}
${ }^{*}$ This work was supported in part by (i) US NSF grants 0915834 and 1117297, (ii) projects GRF 4169/09, 4166/10, 4165/11 from HKRGC, and (iii) the WCU (World Class University) program under the National Research Foundation of Korea, and funded by the Ministry of Education, Science and Technology of Korea (Project No: R31-30007).
\end{abstract}

Permission to make digital or hard copies of all or part of this work for personal or classroom use is granted without fee provided that copies are not made or distributed for profit or commercial advantage and that copies bear this notice and the full citation on the first page. To copy otherwise, to republish, to post on servers or to redistribute to lists, requires prior specific permission and/or a fee. Articles from this volume were invited to present their results at The 38th International Conference on Very Large Data Bases, August 27th - 31st 2012, Istanbul, Turkey.

Proceedings of the VLDB Endowment, Vol. 5, No. 11

Copyright 2012 VLDB Endowment $2150-8097 / 12 / 07 \ldots \$ 10.00$.

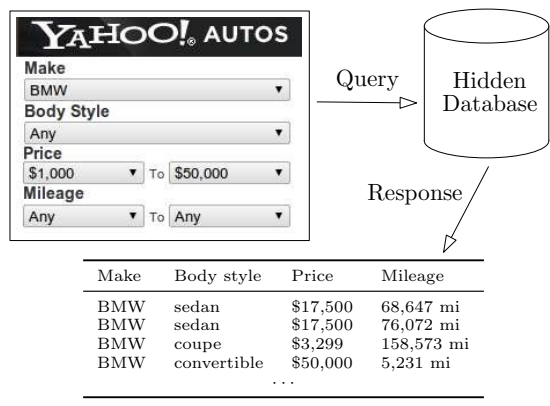

Figure 1: Form-based querying of a hidden database

the current technology, and therefore, are usually referred to as hidden databases.

Consider, for example, Yahoo! Autos (autos.yahoo.com), a popular website for online trading of automobiles. A potential buyer specifies her/his filtering criteria through a form as illustrated in Figure 1. The query is submitted to the system, which runs it against the back-end database, and returns the result to the user. What makes it non-trivial (for a search engine) to crawl the database is that, setting all search criteria to ANY does not accomplish the task. The reason is that a system typically limits the number $k$ of tuples returned ( $k=1000$ for Yahoo! Autos, at the time this paper was written), and that repeating the same query may not retrieve new tuples, i.e., the same $k$ tuples may always be returned.

The ability of crawling a hidden database comes with the appealing promise of enabling virtually any form of processing on the database's content. The challenge, however, is clear: how to obtain all the tuples, given that the system limits the number of return tuples for each query? A naive solution is to issue a query for every single location in the data space (e.g., in Figure 1, the data space is the Cartesian product ${ }^{1}$ of the domains of MAKE, BODY STYLE, PRICE, and Mileage), but the number of queries needed can obviously be prohibitive. This gives rise to an interesting problem, as we define in the next subsection, where the objective is to minimize the number of queries.

\subsection{Problem Definitions}

We consider that the data space $\mathbb{D}$ has $d$ attributes $A_{1}, \ldots, A_{d}$, each of which has a discrete domain. Specifically, denote by $\operatorname{dom}\left(A_{i}\right)$ the domain of $A_{i}$ for each $i \in[1, d]$; then, $\mathbb{D}$ is the Cartesian product of $\operatorname{dom}\left(A_{1}\right), \ldots, \operatorname{dom}\left(A_{d}\right)$. We refer to each element

\footnotetext{
${ }^{1}$ While one may leverage knowledge of attribute dependencies e.g., BMW does not sell trucks in the US - to prune the data space into a subset of the Cartesian product, the subset is often still too large to enumerate.
} 
of the Cartesian product as a point in $\mathbb{D}$, i.e., a point is a possible combination of values of all dimensions.

Depending on whether there is a total ordering on $\operatorname{dom}\left(A_{i}\right)$ or not, we call $A_{i}$ a numeric or categorical attribute, respectively. Our discussion distinguishes three types of $\mathbb{D}$ :

- Numeric: all the $d$ attributes of $\mathbb{D}$ are numeric.

- Categorical: all the $d$ attributes are categorical. In this case, we use $U_{i}$ to represent the size of $\operatorname{dom}\left(A_{i}\right)$, i.e., how many distinct values there are in $\operatorname{dom}\left(A_{i}\right)$.

- Mixed: the first cat $\in[1, d-1]$ attributes $A_{1}, \ldots, A_{\text {cat }}$ are categorical, whereas the other $d-c a t$ attributes are numeric. Similar to before, let $U_{i}=\left|\operatorname{dom}\left(A_{i}\right)\right|$ for each $i \in[1, \mathrm{cat}]$.

To facilitate presentation, we consider that the domain of a numeric $A_{i}$ to be the set of all integers, whereas that of a categorical $A_{i}$ to be the set of integers from 1 to $U_{i}$. Keep in mind, however, that the ordering of these values is irrelevant to a categorical $A_{i}$.

Let $D$ be the hidden database of a server with each element of $D$ being a point in $\mathbb{D}$. To avoid ambiguity, we will always refer to elements of $D$ as tuples. $D$ is a bag (i.e., a multi-set), that is, it may contain identical tuples.

The server supports queries on $D$. As shown in Figure 1, each query specifies a predicate on each attribute. Specifically, if $A_{i}$ is numeric, the predicate is a range condition in the form of

$$
A_{i} \in[x, y]
$$

where $[x, y]$ is an interval in $\operatorname{dom}\left(A_{i}\right)$. On the other hand, for a categorical $A_{i}$, the predicate is:

$$
A_{i}=x
$$

where $x$ is either a value in $\operatorname{dom}\left(A_{i}\right)$ or a wildcard $\star$. In particular, a predicate $A_{i}=\star$ means that $A_{i}$ can be an arbitrary value in $\operatorname{dom}\left(A_{i}\right)$, i.e., capturing BODY STYLE $=$ ANY in Figure 1. Note that if a hidden database server only allows single-value predicates (i.e., no range-condition support) on a numeric attribute, then we can simply consider the attribute as categorical.

Given a query $q$, denote by $q(D)$ the bag of tuples in $D$ qualifying all the predicates of $q$. The server does not necessarily return the entire $q(D)$ - it does so only when $q(D)$ is small. Formally, the response of the server is:

- if $|q(D)| \leq k$ : the entire $q(D)$ is returned. In this case, we say that $q$ is resolved.

- Otherwise: only $k$ tuples $^{2}$ in $q(D)$ are returned, together with a signal indicating that $q(D)$ still has other tuples. In this case, we say that $q$ overflows.

The value of $k$ is a system parameter (e.g., $k=1000$ for Yahoo! Autos, as mentioned earlier). It is important to note that, in case a query $q$ overflows, repeatedly issuing the same $q$ may always get the same response from the server, and does not help to obtain the other tuples in $q(D)$.

The problem addressed by this paper is:

Problem 1. (Hidden Database Crawling) Retrieve the entire $D$ while minimizing the number of queries.

Recall that $D$ is a bag, i.e., it may have duplicate tuples. We require that no point in the data space $\mathbb{D}$ have more than $k$ tuples in

\footnotetext{
${ }^{2}$ In practice, these are usually the $k$ tuples that have the highest priorities (e.g., according to a ranking function) among all the tuples qualifying the query.
}

$D$. Otherwise, Problem 1 has no solution at all. To see this, consider the existence of $k+1$ tuples $t_{1}, \ldots, t_{k+1}$ in $D$, all of which are equivalent to a point $p \in \mathbb{D}$. Then, whenever $p$ satisfies a query, the server can always choose to leave $t_{k+1}$ out of its response, making it impossible for any algorithm to extract the entire $D$. Note that, in Yahoo! Autos, the previous requirement essentially states that there cannot be $k=1000$ vehicles in the database having exactly the same values on all attributes - an assumption that is fairly realistic.

As mentioned in Problem 1, the cost of an algorithm is the number of queries issued. This metric is motivated by the fact that, most systems have a control on how many queries can be submitted by the same IP address within a period of time (e.g., a day). Therefore, a crawler must minimize the number of queries to get the task done, besides bringing the burden of the server to the lowest level.

We will use $n$ to denote the number of tuples in $D$. It is clear that the number of queries needed to extract the entire $D$ is at least $n / k$. Of course, this ideal cost may not always be possible. Hence, the central (technical) questions to be answered are two-fold. First, on the upper bound side, how to solve Problem 1 by performing only a small number of queries even in the worst case? Second, on the lower bound side, how many queries are compulsory for solving the problem in the worst case?

\subsection{Our Results}

This paper presents a systematic study of hidden database crawling as defined in Problem 1. At a high level, our first contribution is a set of algorithms that are both provably fast in the worst case, and efficient on practical data. Our second contribution is a set of lowerbound results establishing the hardness of the problem. These results make explicit how the hardness is affected by the underlying factors, and thus reveal valuable insights into the characteristics of the problem. Furthermore, the lower bounds also prove that our algorithms are already optimal asymptotically, i.e., they cannot be improved by more than a constant factor.

Our first main result is:

THEOREM 1. There is an algorithm for solving Problem 1 whose cost is:

- $O\left(d \cdot \frac{n}{k}\right)$ when $\mathbb{D}$ is numeric;

- at most $U_{1}$ when $\mathbb{D}$ is categorical and cat $=1$ (i.e., there is only one categorical attribute);

- at most $\frac{n}{k} \cdot \sum_{i=1}^{d} \min \left\{U_{i}, \frac{n}{k}\right\}+\sum_{i=1}^{d} U_{i}$ when $\mathbb{D}$ is categorical and cat $>1$;

- at most $U_{1}+O\left(d \cdot \frac{n}{k}\right)$ when $\mathbb{D}$ is mixed and cat $=1$;

- otherwise (i.e., $\mathbb{D}$ is mixed and cat $>1$ ): at most

$$
\frac{n}{k} \cdot \sum_{i=1}^{c a t} \min \left\{U_{i}, \frac{n}{k}\right\}+\sum_{i=1}^{c a t} U_{i}+O\left((d-c a t) \frac{n}{k}\right) .
$$

The above can be conveniently understood as follows: our algorithm pays an (additive) cost of $O(n / k)$ for each numeric attribute $A_{i}$, whereas it pays $\frac{n}{k} \cdot \min \left\{U_{i}, \frac{n}{k}\right\}+U_{i}$ for each categorical $A_{i}$. The only exception is when cat $=1$ : in this scenario, we pay merely $U_{1}$ for the (only) categorical attribute $A_{1}$. Notice that the cost on each numeric attribute is irrelevant to its domain size.

Our second main result complements the preceding one:

THEOREM 2. None of the results in Theorem 1 can be improved by more than a constant factor in the worst case.

Besides establishing the optimality of our upper bounds in Theorem 1, Theorem 2 has its own interesting implications. First, it indicates the unfortunate fact that, for all types of $\mathbb{D}$, the best achievable query time (in the worst case) is much higher than the ideal 
cost of $n / k$ (nevertheless, Theorem 1 suggests that we can achieve this cost asymptotically when $d$ is a constant and all attributes are numeric). Second, as the number cat of categorical attributes increases from 1 to 2 , the discrepancy of the time complexities in Theorem 1 is not an artifact, but rather, it is due to an inherent leap in the hardness of the problem (this is true regardless of the number of numeric attributes). That is, while we pay only $O\left(U_{1}\right)$ extra queries for the (sole) categorical attribute when $c a t=1$, as cat grows to 2 and onwards, the cost paid (by any algorithm) for each categorical $A_{i}$ has an extra term of $\frac{n}{k} \min \left\{U_{i}, \frac{n}{k}\right\}$. Given that the term is multiplicative, this finding implies (perhaps surprisingly) that, in the worst case, it may be infeasible to crawl a hidden database with a large size $n$, and at least 2 categorical attributes such that at least one of them has a large domain.

We have performed extensive experiments to evaluate the efficiency of the proposed algorithms on real datasets, and demonstrate that they demand significantly fewer queries than alternative solutions. Our experimentation also reveals that the number of queries needed to crawl a hidden database may be far less than previously thought; for example, for $k=1000$, around 200 queries already suffice for crawling a dataset containing 69,768 tuples from the hidden database at Yahoo! Autos. This phenomenon suggests that, for a search engine, crawling a hidden database may no longer be a goal of tomorrow, whereas for a data provider, permitting an engine to crawl its database is not expected to impose a heavy toll on its workload.

\subsection{Practical Remarks}

Domain values. In our problem definition, the crawler should know the domains of the categorical attributes (note that this issue is irrelevant to numerical attributes, whose domains can always be considered to be $(-\infty, \infty))$. For some websites, the domains of all attributes are explicitly provided such that our algorithms can be applied immediately. For example, this is the case for Yahoo! Autos, where all the values of, say, MAKE can be seen from the pull-down menu of its query interface. For other websites, before using our technique, the crawler needs to first discover the domain values of categorical attributes. Domain discovery has been studied in [15], and can be accomplished with a number of effective algorithms.

Attribute dependency. As mentioned in the above discussions, because of attribute dependencies in a practical hidden database, not all the points in the data space $\mathbb{D}$ can contain a tuple. For example, with proper external knowledge of the dependency between MAKE and BODY STYLE, one does not need to explore points with MAKE = BMW and BODY STYLE = TRUCK. While knowledge of attribute dependencies is not considered in this paper, please note that our upper bound results still hold even in scenarios where attribute dependencies exist. In other words, even if our algorithm may issue some unnecessary queries, the number of queries needed can nonetheless still be limited under the claimed bounds, as is exactly the merit of Theorem 1. In practice, there is an obvious heuristic for adapting our algorithm to account for attribute dependencies: the crawler issues a query demanded by our algorithm only if the query covers at least one valid point in $\mathbb{D}$ (according to the crawler's dependency knowledge). The query cost can only go down, i.e., still guaranteed to be below our upper bounds.

Acquisition of knowledge about attribute dependencies requires dedicated efforts to analyze the hidden database at a particular website - efforts that obviously cannot be afforded by the crawler for all websites. The implication is thus that the crawler may not be aware of the latent attribute dependencies at many of the websites being crawled. This is where our lower bound results come into place: they indicate the ultimate worst-case efficiency that the crawler can possibly achieve in these environments: a piece of information vital for the crawler's design.

\subsection{Previous Work}

A significant body of research has been carried out on how to extract, integrate, and analyze data from the deep web, a general term referring to the entire collection of online information unreachable by search engines (including, but not limited to, hidden databases). As explained below, however, the issues that have been addressed by the existing work are all orthogonal to this paper.

Most relevant to our work are the previous studies on crawling hidden text-based $[1,5,18,20]$ and structured $[2,7,16,17,19]$ databases. The focus of those studies is how to formulate queries to retrieve meaningful results. More specifically, the primary challenge in $[1,5,18,20]$, where the query interface is a keyword-based (Google-like) form, is to discover legitimate query keywords. On the other hand, the main objective in $[2,7,16,17,19]$, where the query interface is an HTML-form like the one in Figure 1, is to expose combinations of input values suitable for filling in the form. In this paper, we are not concerned with mining effective queries, but instead, attack directly how to acquire a complete hidden database with the smallest number of queries (see Problem 1).

Also relevant is the literature of data analytics in deep web. In this vein, a main stream is to investigate how sampling can be deployed to perform, for example, content summary generation $[8,14]$, top- $k$ retrieval [6], aggregate estimation [9], measurement of various metrics of search engines $[3,4]$, and so on. The crawling techniques proposed in this paper aim at enabling a much broader class of applications (e.g., virtually any query on the database, as described earlier), which otherwise would not be possible if only a sample of the hidden database could be obtained.

It is worth mentioning that, there has been considerable research on other problems related to the deep web, which, however, are only remotely related to our work. While a complete survey is out of the scope of this paper, entry points for further reading can be found in $[10,21]$ on parsing and understanding web interfaces, in [13] on attribute mapping across different interfaces, and in [11,12] for integrating the query interfaces of multiple hidden databases.

\section{NUMERICAL ATTRIBUTES}

This section will explain how to solve Problem 1 when the data space $\mathbb{D}$ is numeric. In Section 2.1, we first define some atomic operators, and present an algorithm that is intuitive, but has no attractive performance bounds. Then, in Sections 2.2 and 2.3, we present another algorithm to achieve the optimal performance.

\subsection{Basic Operations and Baseline Algorithm}

Recall that, in a numeric $\mathbb{D}$, the predicate of a query $q$ on each attribute is a range condition. Thus, $q$ can be regarded as a $d$ dimensional (axis-parallel) rectangle, such that its result $q(D)$ consists of the tuples of $D$ covered by that rectangle. If the predicate of $q$ on attribute $A_{i}(i \in[1, d])$ is $A_{i} \in\left[x_{1}, x_{2}\right]$, we say that $\left[x_{1}, x_{2}\right]$ is the extent of the rectangle of $q$ along $A_{i}$. Henceforth, we may use symbol $q$ to refer to its rectangle also, when no ambiguity can be caused. Clearly, settling Problem 1 is equivalent to determining the entire $q(D)$ where $q$ is the rectangle covering the whole $\mathbb{D}$.

Split. A fundamental idea to extract all the tuples in $q(D)$ is to refine $q$ into a set $S$ of smaller rectangles, such that each rectangle $q^{\prime} \in S$ can be resolved (i.e., $q^{\prime}(D)$ has at most $k$ tuples). Note that this always happens as long as rectangle $q^{\prime}$ is sufficiently small in the extreme case, when $q^{\prime}$ has degenerated into a point in $\mathbb{D}$, the query $q^{\prime}$ is definitely resolved (otherwise, there would be at least 


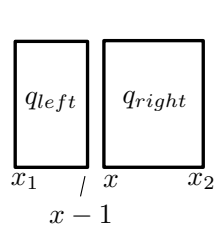

(a) 2-way

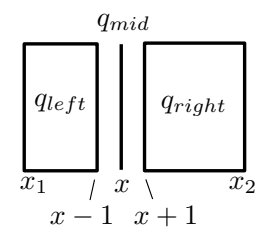

(b) 3-way
Figure 2: Splitting

$k+1$ tuples of $D$ at this point). Therefore, a basic operation in our algorithms for Problem 1 is split, as described next.

Given a rectangle $q$, we may perform two types of splitting, depending on how many rectangles $q$ is divided into:

- 2-way split: Let $\left[x_{1}, x_{2}\right]$ be the extent of $q$ on $A_{i}$ (for some $i \in[1, d]$ ). A 2-way split at a value $x \in\left[x_{1}, x_{2}\right]$ partitions $q$ into rectangles $q_{\text {left }}$ and $q_{\text {right }}$, by dividing the $A_{i}$-extent of $q$ at $x$. Formally, on any attribute other than $A_{i}$, $q_{\text {left }}$ and $q_{\text {right }}$ have the same extents as $q$. Along $A_{i}$, however, the extent $q_{\text {left }}$ is $\left[x_{1}, x-1\right]$, whereas that of $q_{\text {right }}$ is $\left[x, x_{2}\right]$. Figure $2 \mathrm{a}$ illustrates the idea by splitting on the horizontal attribute.

- 3-way split: Let $\left[x_{1}, x_{2}\right]$ be defined as above. A 3-way split at a value $x \in\left[x_{1}, x_{2}\right]$ partitions $q$ into rectangles $q_{l e f t}$, $q_{\text {mid }}$ and $q_{\text {right }}$ as follows. On any attribute other than $A_{i}$, they have the same extent as $q$. Along $A_{i}$, however, the extent of $q_{l e f t}$ is $\left[x_{1}, x-1\right]$, that of $q_{\text {mid }}$ is $[x, x]$, and that of $q_{\text {right }}$ is $\left[x+1, x_{2}\right]$. See Figure $2 \mathrm{~b}$.

In the sequel, a 2-way split will be abbreviated simply as a split. No confusion can arise as long as we always mention 3-way in referring to a 3-way split. The extent of a query $q$ on an attribute $A_{i}$ can become so short that it covers only a single value, in which case we say that $A_{i}$ is exhausted on $q$. For instance, the horizontal attribute is exhausted on $q_{m i d}$ in Figure $2 \mathrm{~b}$. It is easy to see that there is always a non-exhausted attribute on $q$ unless $q$ has degenerated into a point.

Binary shrink. Next, we describe a straightforward algorithm for solving Problem 1, which will serve as the baseline approach for comparison. This algorithm, named binary-shrink, repeatedly performs (2-way) splits until a query is resolved. Specifically, given a rectangle $q$, binary-shrink runs the rectangle (by submitting its corresponding query to the server) and finishes if $q$ is resolved. Otherwise, the algorithm splits $q$ on an attribute $A_{i}$ that has not been exhausted, by cutting the extent $\left[x_{1}, x_{2}\right]$ of $q$ along $A_{i}$ into equally long intervals (i.e., the split is performed at $x=\left\lceil\left(x_{1}+x_{2}\right) / 2\right\rceil$ ). Let $q_{\text {left }}, q_{\text {right }}$ be the queries produced by the split. The algorithm then recurses on $q_{\text {left }}$ and $q_{\text {right }}$, respectively.

Remark. It is obvious that the cost of binary-shrink (i.e., the number of queries issued) depends on the domain sizes of the (numeric) attributes of $\mathbb{D}$, which can be unbounded. In the following subsections, we will improve this algorithm to optimality.

\subsection{One-Dimensional Case}

Before giving our ultimate algorithm for settling Problem 1 of any dimensionality $d$, in this subsection we first explain how it works for $d=1$. This will clarify the rationale behind the algorithm's efficiency, and facilitate our analysis for a general $d$. It is worth mentioning that the presence of only one attribute removes the need to specify the split dimension in describing a split.

Rank-shrink. Our algorithm, named rank-shrink, differs from binary-shrink in two ways. First, when performing a (2-way) split,

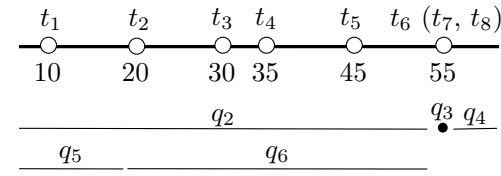

(a) Dataset $D$ and queries

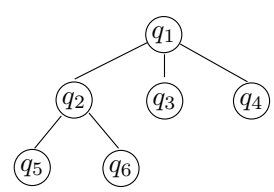

(b) Recursion tree

\section{Figure 3: Illustration of 1d rank-shrink}

instead of cutting the extent of a query $q$ in half, we aim at ensuring that at least $k / 4$ tuples fall in each of the rectangles generated by the split. Such a split, however, may not always be possible, which as we will see can happen if many tuples are identical to each other. Hence, the second difference that rank-shrink makes is to perform a 3 -way split in such a scenario, which gives birth to a query (among the 3 created) that can be immediately resolved.

Formally, given a query $q$, the algorithm eventually returns $q(D)$. It starts by issuing $q$ to the server, which returns a bag $R$ of tuples. If $q$ is resolved, the algorithm terminates by reporting $R$. Otherwise (i.e., $q$ overflows), we sort the tuples of $R$ in ascending order, breaking ties arbitrarily. Let $o$ be the $(k / 2)$-th tuple in the sorted order, with its $A_{1}$-value being $x$. Now, we count the number $c$ of tuples in $R$ identical to $o$ (i.e., $R$ has $c$ tuples with $A_{1}$-value $x$ ), and proceed as follows:

- Case 1: $c \leq k / 4$. Split $q$ at $x$ into $q_{\text {left }}$ and $q_{\text {right }}$, each of which must contain at least $k / 4$ tuples in $R$. To see this for $q_{\text {left }}$ (symmetric reasoning applies to $q_{\text {right }}$ ), note there are at least $k / 2-c \geq k / 4$ tuples of $R$ strictly smaller than $x$, all of which fall in $q_{\text {left }}$. The case for $q_{\text {right }}$ follows in analogy.

- Case 2: $c>k / 4$. Perform a 3-way split on $q$ at $x$. Let $q_{\text {left }}, q_{\text {mid }}$ and $q_{\text {right }}$ be the resulting rectangles (note that, the ordering among them matters; see Section 2.2). Observe that $q_{\text {mid }}$ has degenerated into point $x$, and therefore, can immediately be resolved.

As a technical remark, in Case $2, x$ might be the lower (resp. upper) bound ${ }^{3}$ on the extent of $q$. If this happens, we simply discard $q_{\text {left }}$ (resp. $q_{\text {right }}$ ) as it would have a meaningless extent.

In either case, we are left with at most two queries (i.e., $q_{l e f t}$ and $\left.q_{\text {right }}\right)$ to further process. The algorithm handles each of them recursively in the same manner.

Example. We use the dataset $D$ in Figure 3 a to demonstrate the algorithm. Let $k=4$. The first query is $q_{1}=(-\infty, \infty)$. Suppose that the server responds by returning $R_{1}=\left\{t_{4}, t_{6}, t_{7}, t_{8}\right\}$ and a signal that $q_{1}$ overflows. The $(k / 2)=2$-nd smallest tuple in $R_{1}$ is $t_{6}$ (after random tie breaking), whose value is $x=55$. As $R_{1}$ has $c=3$ tuples with value 55 and $c>k / 4=1$, we perform a 3 -way split on $q_{1}$ at 55, generating $q_{2}=(-\infty, 54], q_{3}=[55,55]$ and $q_{4}=[56, \infty)$. As $q_{3}$ has degenerated into a point, it is resolved immediately, fetching $t_{6}, t_{7}$ and $t_{8}$. These tuples have already been extracted before, but this time they come with an extra fact that no more tuple can exist at point 55 .

Let us look at $q_{2}$. Suppose that the server's response is $R_{2}=$ $\left\{t_{1}, t_{2}, t_{4}, t_{5}\right\}$, plus an overflow signal. Hence, $x=20$ and $c=1$. Thus, a two-way split on $q_{2}$ at 20 creates $q_{5}=(-\infty, 19]$ and $q_{6}=$ [20,54]. Queries $q_{4}, q_{5}$ and $q_{6}$ are all resolved.

Analysis. The lemma below bounds the cost of rank-shrink.

LEMMA 1 . When $d=1$, rank-shrink requires $O(n / k)$ queries.

\footnotetext{
${ }^{3} x$ cannot be both because otherwise $q$ would be a point and there-
} fore could not have overflown. 
PROOF. The main tool used by our proof is a recursion tree $T$ that captures the spawning relationships of the queries performed by rank-shrink. Specifically, each node of $T$ represents a query. Node $u$ is the parent of node $u^{\prime}$ if query $u^{\prime}$ is created by a (2-way or 3-way) split of query $u$. Each internal node thus has 2 or 3 child nodes. Figure $3 \mathrm{~b}$ shows the recursion tree for the queries performed in our earlier example on Figure $3 \mathrm{a}$.

We focus on bounding the number of leaves in $T$ because it dominates the number of internal nodes. Observe that each leaf $v$ corresponds to a disjoint interval in $\operatorname{dom}\left(A_{1}\right)$, due to the way splits are carried out. There are three types of $v$ :

- Type-1: the query represented by $v$ is immediately resolved in a 3-way split (i.e., $q_{m i d}$ in Case 2). The interval of $v$ contains at least $k / 4$ (identical) tuples in $D$.

- Type-2: query $v$ is not type-1, but also covers at least $k / 4$ tuples in $D$.

- Type-3: query $v$ covers less than $k / 4$ tuples in $D$.

For example, among the leaf nodes in Figure 3, $q_{3}$ is of type $1, q_{5}$ and $q_{6}$ are of type 2, and $q_{4}$ is of type 3 .

As the intervals of various leaves cover disjoint bags of tuples, the number of type- 1 and -2 leaves is at most $\frac{n}{k / 4}=4 n / k$. Each leaf of type- 3 must have a sibling in $T$ that is a type-2 leaf (i.e., in Figure 3, such a sibling of $q_{4}$ is $q_{3}$ ). On the other hand, a type-2 leaf has at most 2 siblings. It thus follows that there are at most twice as many type- 3 leaves as type- 2 , i.e., the number of type- 3 leaves is no more than $8 n / k$. This completes the proof.

We remark that the above analysis implies that (quite loosely) $T$ has no more than $4 n / k+8 n / k=12 n / k$ leaves. Thus, there cannot be more than this number of internal nodes in $T$.

\subsection{Rank-Shrink for Higher Dimensionality}

We are now ready to extend rank-shrink to handle any $d>1$. In addition to the ideas exhibited in the preceding subsection, we also apply an inductive approach: converting the $d$-dimensional problem to several $(d-1)$-dimensional ones. Our discussion below assumes that the $(d-1)$-dimensional problem has already been settled by rank-shrink.

Given a query $q$, the algorithm (as in 1d) sets out to solicit the server's response $R$, and finishes if $q$ is resolved. Otherwise, it examines whether $A_{1}$ is exhausted in $q$, i.e., whether the extent of $q$ on $A_{1}$ has only 1 value, say $x$, in $\operatorname{dom}\left(A_{1}\right)$. If so, we can then focus on attributes $A_{2}, \ldots, A_{d}$. This is a $(d-1)$-dimensional version of Problem 1, in the $(d-1)$-dimensional subspace covered by the extents of $q$ on $A_{2}, \ldots, A_{d}$, eliminating $A_{1}$ by fixing it to $x$. Hence, we invoke rank-shrink to solve it.

Consider that $A_{1}$ is not exhausted on $q$. Similar to the $1 \mathrm{~d}$ algorithm, we will split $q$ such that, either every resulting rectangle covers at least $k / 4$ tuples in $R$, or one of them can be immediately solved as a $(d-1)$-dimensional problem. The splitting proceeds exactly as described in Cases 1 and 2 of Section 2. The only difference is that the rectangle $q_{m i d}$ in Case 2 is not a point, but instead, a rectangle on which $A_{1}$ has been exhausted. Hence, $q_{m i d}$ is processed as a $(d-1)$-dimensional problem with rank-shrink.

As with the $1 \mathrm{~d}$ case, the algorithm recurses on $q_{\text {left }}$ and $q_{\text {right }}$ (provided that they have not been discarded for having a meaningless extent on $A_{1}$ ).

Example. We demonstrate the algorithm using the $2 \mathrm{~d}$ dataset in Figure 4 , where $D$ has 10 tuples $t_{1}, \ldots, t_{10}$. Let $k=4$. The first query $q_{1}$ issued covers the entire data space. Suppose that the server responds with $R_{1}=\left\{t_{4}, t_{7}, t_{8}, t_{9}\right\}$ and an overflow signal. We 3-way split $q_{1}$ at $A_{1}=80$ into $q_{2}, q_{3}$ and $q_{4}$, whose

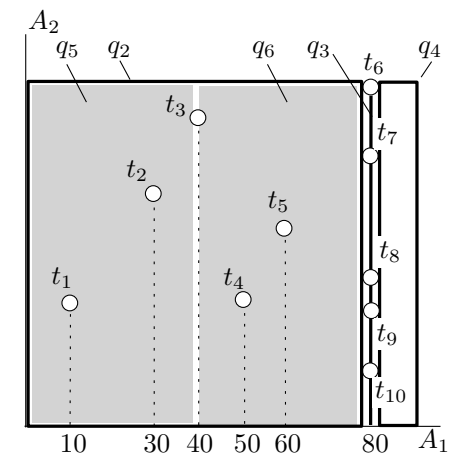

Figure 4: Illustration of 2d rank-shrink

rectangles can be found in Figure 4. Specifically, the $A_{1}$-extents of $q_{2}, q_{3}, q_{4}$ are $(-\infty, 79],[80,80],[81, \infty)$ respectively, while their $A_{2}$-extents are all $(-\infty, \infty)$. Note that $A_{1}$ is exhausted on $q_{2} ;$ alternatively, we can see that $q_{2}$ is equivalent to a $1 \mathrm{~d}$ query on the vertical line $A_{1}=80$. Hence, $q_{2}$ is recursively settled by our $1 \mathrm{~d}$ algorithm (which, as can be verified easily, requires 3 queries).

Suppose that the server's response to $q_{2}$ is $R_{2}=\left\{t_{2}, t_{3}, t_{4}, t_{5}\right\}$ and an overflow signal. Accordingly, $q_{2}$ is split into $q_{5}$ and $q_{6}$ at $A_{1}=40$, whose rectangles are also shown in Figure 4. Finally, $q_{4}, q_{5}$ and $q_{6}$ are all resolved.

Analysis. We have the lemma below for general $d$ :

\section{LEMMA 2. Rank-shrink performs $O(d n / k)$ queries.}

Proof. The case $d=1$ has been proved in Lemma 1. Next, assuming that rank-shrink issues at most $\alpha(d-1) n / k$ queries for solving a $(d-1)$-dimensional problem with $n$ tuples (where $\alpha$ is a positive constant), we will show that the cost is at most $\alpha d n / k$ for dimensionality $d$.

Again, our argument leverages a recursion tree $T$. As before, each node of $T$ is a query, such that node $u$ parents node $u^{\prime}$, if query $u^{\prime}$ was created from splitting $u$. We make a query $v$ a leaf of $T$ as soon as one of the following occurs:

- $v$ is resolved. We associate $v$ with a weight set to 1 .

- $A_{1}$ is exhausted on rectangle $v$. Recall that such a query is solved as a $(d-1)$-dimensional problem. We associate $v$ with a weight, equal to the cost for rank-shrink for that problem.

For our earlier example with Figure 4, the recursion tree $T$ happens to be the same as the one in Figure $3 b$. The difference is that each leaf has a weight. Specifically, the weight of $q_{3}$ is 3 (i.e., the cost of solving the $1 \mathrm{~d}$ query at the vertical line $A_{1}=80$ in Figure 4), and the weights of the other leaves are 1.

The total cost of rank-shrink on the $d$-dimensional problem, therefore, equals the total number of internal nodes in $T$, plus the total weight of all the leaves.

As the $A_{1}$-extents of the leaves' rectangles have no overlap, their rectangles cover disjoint tuples. Let us classify the leaves into types- $1,-2$ and -3 as in the proof of Lemma 1 , by adapting the definition of type- 1 in a straightforward fashion: $v$ is of this type if it is the middle node $q_{\text {mid }}$ from a 3-way split. Each type-3 leaf has weight 1 (as its corresponding query must be resolved). As proved in Lemma 1 , the number of them is no more than $8 n / k$.

Let $v_{1}, \ldots, v_{\beta}$ be all the type- 1 and -2 nodes (i.e., suppose the number of them is $\beta$ ). Assume that node $v_{i}$ contains $n_{i}$ tuples of $D$. It holds that $\sum_{i=1}^{\beta} n_{i} \leq|D|=n$. The weight of $v_{i}$, by our inductive assumption, is at most $\alpha(d-1) n_{i} / k$. Hence, the total weight of all the type- 1 and -2 nodes does not exceed $\alpha(d-1) n / k$. 
The same argument in the proof of Lemma 1 shows that $T$ has less than $12 n / k$ internal nodes. Thus, summarizing the above analysis, the cost of $d$-dimensional rank-shrink is no more than: $\frac{12 n}{k}+\frac{8 n}{k}+\alpha(d-1) \frac{n}{k}=(20+\alpha(d-1)) \frac{n}{k}$. To complete our inductive proof, we want $(20+\alpha(d-1)) \frac{n}{k}$ to be bounded from above by $\alpha d n / k$. This is true for any $\alpha \geq 20$.

Remark. This concludes the proof of the first bullet of Theorem 1 . We point out that when $d$ is fixed value (as is true in practice), the time complexity in Lemma 2 becomes $O(n / k)$, that is, asymptotically matching the trivial lower bound $n / k$. A natural question at this point is, if $d$ is not constant, is there an algorithm that can still guarantee cost $O(n / k)$ ? In Section 4 , we will show that this is impossible.

\section{CATEGORICAL ATTRIBUTES}

We proceed to solve Problem 1 when the data space $\mathbb{D}$ is categorical. Recall that, as mentioned in Section 1.1, the domain $\operatorname{dom}\left(A_{i}\right)$ of the $i$-th attribute $A_{i}$ is the set of integers in $\left[1, U_{i}\right]$ (where $U_{i}=\left|\operatorname{dom}\left(A_{i}\right)\right|$ ), although it should be understood that the ordering of those integers is irrelevant. We will again first (in Section 3.1) clarify some preliminary concepts and give a baseline algorithm, before presenting the proposed solution (in Section 3.2).

\subsection{Data Space Tree and Depth First Search}

Unlike range predicates on numeric attributes, the predicate supported by the server on a categorical attribute $A_{i}(1 \leq i \leq d)$ is an equality constraint of the form $A_{i}=x$, where $x$ is either a value in $\operatorname{dom}\left(A_{i}\right)$ or a wildcard $\star$. This difference prompts us to adopt an alternative approach to attack Problem 1. Instead of performing 2or 3-way splits (as in the numeric case), we instead enumerate the points in $\mathbb{D}$. This idea looks drastic at first glance $-\mathbb{D}$ has a total of $\prod_{i=1}^{d} U_{i}$ points, where $U_{i}$ is the domain size of $A_{i}$. A naive way to enumerate the entire $\mathbb{D}$ is clearly intractable. It turns out, interestingly, that we can significantly reduce the cost from the formidable $\prod_{i=1}^{d} U_{i}$ to only roughly a linear term $\sum_{i=1}^{d} U_{i}$.

Data space tree. Let us start by arranging all the points of $\mathbb{D}$ into a tree $\mathbb{T}$, which we refer to as the data space tree. Each node $u$ of $\mathbb{T}$ represents a subspace enclosing all the points of $\mathbb{D}$ satisfying a condition like:

$$
A_{1}=c_{1}, \ldots, A_{\ell}=c_{\ell}, A_{\ell+1}=\star, \ldots, A_{d}=\star
$$

where $\ell$ is an integer in $[0, d]$ and $c_{1}, \ldots, c_{\ell}$ are not wildcards. We say that $u$ is at level $\ell$. We will refer to the condition of $u$ as query $(u)$ because the condition obviously corresponds to a query that can be submitted to the server.

If we look at a point $\left(c_{1}, \ldots, c_{d}\right)$ in $\mathbb{D}$ as a string concatenating all its coordinates from the first to the $d$-th attribute, $\mathbb{T}$ can be regarded as a trie on all the $\prod_{i=1}^{d} U_{i}$ strings. Formally, the root of $\mathbb{D}$ is at level 0 , and thus represents the entire $\mathbb{D}$ (equivalently, the condition of the root specifies a wildcard on all dimensions). In general, if $u$ is a level- $\ell$ node $(0 \leq \ell \leq d-1)$, it has $U_{\ell+1}$ child nodes of level $\ell+1$. The $i$-th $\left(1 \leq i \leq U_{\ell+1}\right)$ child $v$ of $u$ is such that, query $(v)$ agrees with query $(u)$ on all dimensions, except $A_{\ell+1}$ on which query $(v)$ specifies $A_{\ell+1}=i$. That is, $v$ refines $u$ on $A_{\ell+1}$ by setting this attribute to $i$. Each leaf of $\mathbb{T}$ is at level $d$ and represents a distinct point in $\mathbb{D}$.

To illustrate, Figure 5a shows a dataset $D$ with 10 tuples $t_{1}, \ldots, t_{10}$ in a $2 \mathrm{~d}$ space $\mathbb{D}$ where each dimension has domain size 4. Figure $5 b$ demonstrates the data space tree $\mathbb{T}$ (the subtrees of nodes $u_{3}$ and $u_{4}$ are omitted for simplicity). Node $u_{1}$, for instance, is associated with a query $\left(u_{1}\right)$ that has predicates $A_{1}=\star$ and

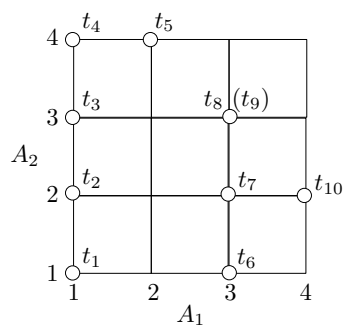

(a) A categorical dataset

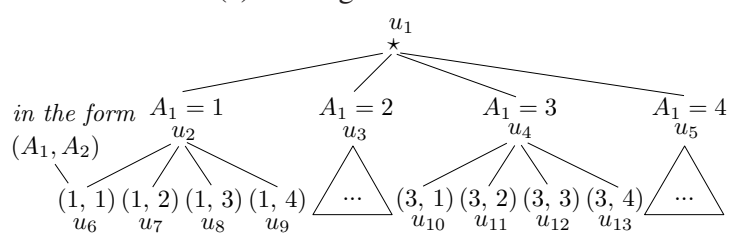

(b) The data space tree

Figure 5: Illustration of categorical algorithms

$A_{2}=\star$, whereas query $\left(u_{2}\right)$ has predicates $A_{1}=1$ and $A_{2}=\star$, and query $\left(u_{7}\right)$ has $A_{1}=1$ and $A_{2}=2$.

The following lemma presents a fact that will be useful later.

Lemma 3. Let $u, v$ be two nodes at the same level of $\mathbb{T}$. No tuple of $D$ can satisfy query $(u)$ and query $(v)$ at the same time.

PROOF. Let the level be $\ell$. The conditions in query $(u)$ and query $(v)$ differ in their predicates on at least one attribute $A_{i}$ for some $i \in[1, \ell]$. No tuple can satisfy those predicates simultaneously.

Depth first search (DFS). We now describe an algorithm, named $D F S$, that serves as the baseline approach. The algorithm simply traverses $\mathbb{T}$ in a depth-first manner. For each node $u$ in $\mathbb{T}$, it sends query $(u)$ to the server, and acquires the bag $R$ of tuples returned. As a pruning rule, if query $(u)$ is resolved, the subtree of $u$ no longer needs to be explored, as all the tuples in the subtree are already in $R$. If, on the other hand, query $(u)$ overflows, the algorithm processes each child of $u$ in the same manner.

Suppose $k=3$. On the input of Figure 5a, DFS examines the nodes in the order $u_{1}, u_{2}, u_{6}, u_{7}, \ldots$ To see an example of pruning, consider the moment when $D F S$ is at $u_{3}$. Since query $\left(u_{3}\right)$ is resolved (the query has predicates $A_{1}=2$ and $A_{2}=\star$, and returns only $t_{5}$ ), the subtree of $u_{3}$ can thus be eliminated. It can be verified that $D F S$ eventually visits all of $u_{1}, \ldots, u_{13}$.

Remark. Not surprisingly, DFS incurs expensive query cost in the worst case. We omit its analysis because it is tedious, and yet this algorithm is not the one advocated in this paper. In the next subsection, we give a better algorithm with the optimal performance.

\subsection{Algorithm Slice-Cover}

Slice query. We say that a query $q$ is a slice query if its predicates have the form:

$$
\ldots, A_{i-1}=\star, A_{i}=c, A_{i+1}=\star, \ldots
$$

where $c$ is a value in $\operatorname{dom}\left(A_{i}\right)$. Namely, the query has a wildcard predicate on all but one attribute $A_{i}$ for some $i \in[1, d]$. We use the notation $A_{i}=c$ to uniquely refer to a slice query. Clearly, varying $c$ in $\operatorname{dom}\left(A_{i}\right)$ defines $U_{i}$ slice queries, such that the total number of slice queries of all dimensions is $\sum_{i=1}^{d} U_{i}$. In the example of Figure $5 \mathrm{a}$, there are totally 8 slice queries.

Slice-cover. Now we describe an algorithm, named slice-cover, for solving Problem 1. The algorithm runs in two phases. In the 


\begin{tabular}{c|cccc} 
constant $c$ & 1 & 2 & 3 & 4 \\
\hline$A_{1}=$ & ovflow & $\left\{t_{5}\right\}$ & ovflow & $\left\{t_{10}\right\}$ \\
$A_{2}=$ & $\left\{t_{1}, t_{6}\right\}$ & $\left\{t_{2}, t_{7}, t_{10}\right\}$ & $\left\{t_{3}, t_{8}, t_{9}\right\}$ & $\left\{t_{4}, t_{5}\right\}$
\end{tabular}

Figure 6: Lookup table of slice queries $(k=3)$

preprocessing phase, we simply submit every slice query to the server, and record its response locally in a lookup table as follows. If a slice query $q$ is resolved, its result $q(D)$ (which has at most $k$ tuples) is entered in the table, whereas if $q$ overflows, we remember nothing but a bit indicating that $|q(D)|>k$. Figure 6 presents the contents of the lookup table for the example in Figure 5, assuming $k=3$.

The second phase of slice-cover executes an algorithm, named extended-DFS, by supplying the root of the data space tree $\mathbb{T}$ as the parameter. In general, given a node $u$ in $\mathbb{T}$, extended-DFS returns all the tuples in $D$ satisfying query $(u)$ (hence, setting $u$ to the root of $\mathbb{T}$ settles Problem 1). Extended-DFS also performs a depthfirst traversal of $\mathbb{T}$ (similar to the DFS algorithm of Section 3.1), but leverages the contents of the lookup table to boost the pruning effectiveness.

Without loss of generality, suppose that $u$ is at level $\ell$ (for some $\ell \in[0, d])$. Extended-DFS starts by sending query $(u)$ to the server. If query $(u)$ is resolved, the algorithm ends by reporting the result received from the server, because the subtree of $u$ can be eliminated (as explained in Section 3.1 for DFS). Next, we focus on the more interesting case where query $(u)$ overflows, implying that $u$ is an internal node of $\mathbb{T}$.

As with DFS, extended-DFS may access each child node $v$ of $u$. However, before doing so, it attempts to answer query $(v)$ locally using the lookup table (i.e., without bothering the server with another query). To explain, recall that $v$, which is at level $\ell+1$, is associated with a query $(v)$ that refines query $(u)$. That is, query $(v)$ inherits the predicates of query $(u)$ on all attributes, except $A_{\ell+1}$ on which query $(v)$ has a predicate, say, $A_{\ell+1}=c$ for some $c \in \operatorname{dom}\left(A_{\ell+1}\right)$.

Let us observe that the result of query $(v)$ is completely contained in the result of the slice query $A_{\ell+1}=c$. Remember that the server's response, say $R$, to the slice query is already available in our lookup table. Therefore, we fetch $R$ from the table (at no cost), and see whether the slice query was resolved. If yes, query $(v)$ can be accurately answered by returning the tuples in $R$ satisfying query $(v)$, in which case the subtree of $v$ does not need to be explored further. If, on the other hand, $R$ shows that the slice query overflew, extended-DFS recursively processes $v$ in the same manner.

Example. We illustrate extended-DFS using the example of Figure 5 . Let $k=3$. The lookup table output by the preprocessing stage is in Figure 6.

The algorithm starts with query $\left(u_{1}\right)$, where $u_{1}$ is the root of $\mathbb{T}$ (Figure $5 \mathrm{~b}$ ). Even without sending query $\left(u_{1}\right)$ to the server, we know that it overflows for sure, because at least one slice query overflew in preprocessing, as is clear from the (lookup) table. Focusing on the first child $u_{2}$ of $u_{1}$, extended-DFS inspects the table to decide whether query $\left(u_{2}\right)$ can be answered locally. The inspection examines slice query $A_{1}=1$, i.e., the predicate by which query $\left(u_{2}\right)$ refines query $\left(u_{1}\right)$. The table indicates that the slice query overflew. Hence, we recursively apply extended-DFS on $u_{2}$.

At $u_{2}$, the algorithm checks the table as to whether query $\left(u_{6}\right)$ can be answered locally. This time, we focus on slice query $A_{2}=1$ (i.e., the extra predicate in query $\left(u_{6}\right)$ compared to query $\left(u_{2}\right)$ ), which turns out to be resolved. Hence, query $\left(u_{6}\right)$ is directly answered from the result $\left\{t_{1}, t_{6}\right\}$ of the slice query (i.e., returning only $t_{1}$, as $t_{6}$ does not qualify query $\left.\left(u_{6}\right)\right)$. Thus, extended-DFS does not recurse into $u_{6}$. Similarly, query $\left(u_{7}\right)$, query $\left(u_{8}\right)$ and query $\left(u_{9}\right)$ can all be answered locally.

We now backtrack to the root of $u_{1}$, and turn attention to the second child $u_{3}$ of $u_{1}$. A lookup is carried out to see whether query $\left(u_{3}\right)$ can be acquired from the table. The answer is yes (using the result of slice query $A_{1}=2$ ). Hence, the subtree of $u_{3}$ is not explored further. The rest of the execution proceeds in the same manner. Overall, besides $u_{1}$, extended-DFS is also (recursively) invoked on $u_{2}$ and $u_{4}$. No query is ever issued to the server in the entire process.

Heuristic. Next, we give a heuristic that does not affect the worstcase cost of slice-cover, but can improve its performance on real data. The motivating rationale is that, some slice queries executed in the pre-processing phase may not eventually be needed. In any case, even if a slice query does need to be consulted by the algorithm, there is no harm to run the query at the first time such a need arises, and register the server's response in the lookup table. If the slice query is consulted for a second time, (as before) the query does not need to be re-issued, for its result is already available in the table. This allows us to get rid of the entire preprocessing phase. We refer to the algorithm equipped with this heuristic as lazy-slicecover.

Analysis. We bound the performance of slice-cover with the following result, which also applies to lazy-slice-cover as it does not require any more query than slice-cover.

LEMMA 4. If $d>1$, slice-cover performs at most $\sum_{i=1}^{d} U_{i}+$ $\frac{n}{k} \cdot \sum_{i=1}^{d} \min \left\{U_{i}, \frac{n}{k}\right\}$ queries. If $d=1$, the number of queries is

PROOF. For $d=1$, slice-cover terminates right after the preprocessing phase, and hence, issues $U_{1}$ queries. For $d \geq 2$, the preprocessing phase obviously issues $\sum_{i=1}^{d} U_{i}$ queries. Next, we will show that extended-DFS incurs cost at most $\frac{n}{k} \cdot \sum_{i=1}^{d} \min \left\{U_{i}, \frac{n}{k}\right\}$.

Let $T$ be the nodes of $\mathbb{T}$ on which extended-DFS is invoked (i.e., $T$ includes the root of $\mathbb{T}$, and the nodes extended-DFS recurses into). For instance, on the example of Figure 5, our earlier discussion showed that $T$ includes nodes $u_{1}, u_{2}$ and $u_{4}$. The number of nodes in $T$ is an upper bound of the number of queries extendedDFS performs.

For each $i \in[1, d]$, let $S_{i}$ be the set of level- $i$ nodes in $T$. The following analysis will prove that $\left|S_{i}\right| \leq \frac{n}{k} \cdot \min \left\{U_{i}, \frac{n}{k}\right\}$, which is enough to complete the proof. To bound $\left|S_{i}\right|$, we consider $S_{i-1}$ (i.e., one level closer to the root). We will show:

- Fact 1: at most $n / k$ nodes in $S_{i-1}$ are internal in $T$.

- Fact 2: each internal node in $S_{i-1}$ can have at most $\min \left\{U_{i}, \frac{n}{k}\right\}$ child nodes in $T$.

Combining both facts gives the desired upper bound $\frac{n}{k}$. $\min \left\{U_{i}, \frac{n}{k}\right\}$ on $\left|S_{i}\right|$.

Proof of Fact 1. For a node $u \in S_{i-1}$, denote by $n_{u}$ the number of tuples in $D$ satisfying query $(u)$. It follows from Lemma 3 that $\sum_{u \in S_{i-1}} n_{u} \leq|D|=n$.

On the other hand, as $u$ is an internal node in $T, n_{u}>k$. Otherwise, query $(u)$ would have been resolved, in which case the subtree of $u$ should have been pruned in extended-DFS, contradicting $u$ being an internal node. This, together with $\sum_{u \in S_{i-1}} n_{u} \leq n$, proves that $S_{i-1}$ has no more than $n / k$ internal nodes.

Proof of Fact 2. For each node $u \in S_{i-1}$, let children $(u)$ be the set of child nodes of $u$ in $\mathbb{T}$. By the way $\mathbb{T}$ is defined, $\mid$ children $(u) \mid=U_{i}$. As $u$ cannot have more child nodes in $T$ 
than in $\mathbb{T}$, Fact 2 holds if $U_{i} \leq n / k$. The rest of the proof assumes $U_{i}>n / k$.

For each node $v \in \operatorname{children}(u)$, query $(v)$ refines query $(u)$ by replacing the predicate on $A_{i}$ (which is $A_{i}=\star$ in query $(u)$ ) with $A_{i}=c$ for some $c \in \operatorname{dom}\left(U_{i}\right)$. Furthermore, each $v$ specifies a different $c$. Extended-DFS guarantees that $v$ should not be accessed (and hence, should not belong to $T$ ) if the slice query $A_{i}=c$ is resolved. In other words, if we denote by $Q$ the set of slice queries of the form $A_{i}=c$ (i.e., $|Q|=U_{i}$ ), the number of child nodes of $u$ in $T$ is at most the number of queries in $Q$ that overflow.

Clearly, no tuple of $D$ can satisfy both slice queries $A_{i}=c_{1}$ and $A_{i}=c_{2}$ as long as $c_{1} \neq c_{2}$. In other words, the sum of $|q(D)|$ (i.e., the number of tuples satisfying $q$ ) of all $q \in Q$ is at most $n$. On the other hand, $|q(D)| \geq k$ if $q$ overflows. Thus, the number of overflowing queries in $Q$ is at most $n / k$.

Remark. Lemma 4 establishes the second and third bullets of Theorem 1. As we will see in the next section, the upper bounds in the lemma cannot be improved by more than a constant factor in the worst case.

\section{LOWER BOUND RESULTS}

This section turns away from upper bounds, and focuses on the hardness of Problem 1. In Section 4.1 (4.2), we will give asymptotic lower bounds on how many queries are needed for settling the problem, when the data space $\mathbb{D}$ is numeric (categorical).

\subsection{Numeric Attributes}

The objective of this subsection is to establish:

THEOREM 3. Let $k, d, m$ be arbitrary positive integers such that $d \leq k$. There is a dataset $D$ (in a numeric data space) with $n=m(k+d)$ tuples such that, any algorithm must use at least dm queries to solve Problem 1 on $D$.

It is, therefore, impossible to improve our algorithm rank-shrink (see Lemma 2) by more than a constant factor in the worst case, as shown below:

COROLlARY 1. In a numeric data space, no algorithm can guarantee solving Problem 1 with o $(d n / k)$ queries.

PROOF. If there existed such an algorithm, let us use it on the inputs in Theorem 3. The cost is $o(d n / k)=o(d m(k+d) / k)$ which, due to $d \leq k$, is $o(d m)$, causing a contradiction.

We now proceed to prove Theorem 3, using a hard dataset $D$ as illustrated in Figure 7. The domain of each attribute is the set of integers from 1 to $m+1$, namely, $\mathbb{D}=[1, m+1]^{d}$. $D$ has $m$ groups of $d+k$ tuples. Specifically, the $i$-th $(1 \leq i \leq m)$ group has $k$ tuples at the point $(i, \ldots, i)$, taking value $i$ on all attributes. We call them diagonal tuples. Furthermore, for each $j \in[1, d]$, Group $i$ also has a tuple that takes value $i+1$ on attribute $A_{j}$, and $i$ on all other attributes. Such a tuple is referred to as a non-diagonal tuple. Overall, $D$ has $\mathrm{km}$ diagonal and $d m$ non-diagonal tuples.

Let $S$ be the set of $d m$ points in $\mathbb{D}$ that are equivalent to the $d m$ non-diagonal tuples in $D$, respectively (i.e., each point in $S$ corresponds to a distinct non-diagonal tuple). As explained in Section 2.1, each query can be regarded as an axis-parallel rectangle in $\mathbb{D}$. With this correspondence in mind, we observe the following for any algorithm that correctly solves Problem 1 on $D$ :

LEMMA 5. When the algorithm terminates, each point in $S$ must be covered by a distinct resolved query already performed.

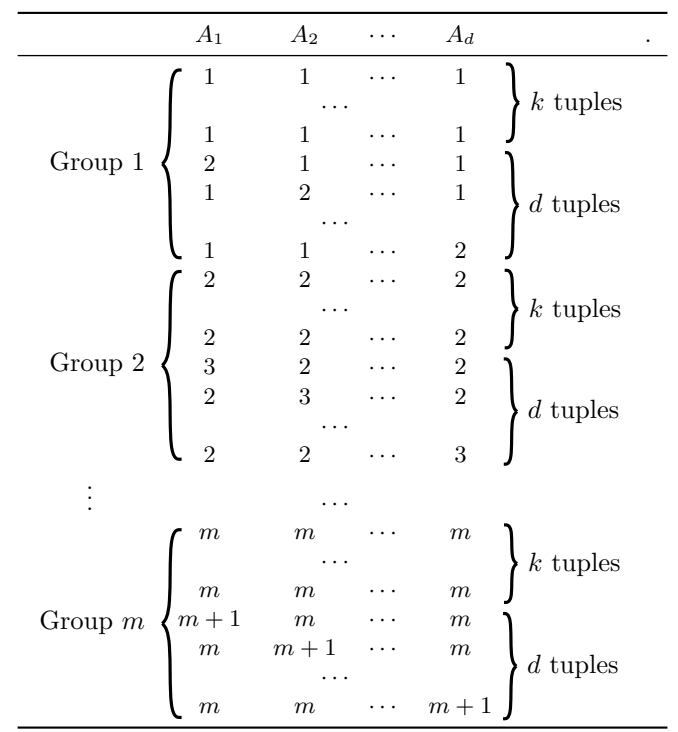

Figure 7: A hard numeric dataset

PROOF. Every point $p \in S$ must be covered by a resolved query. Otherwise, $p$ is either never covered by any query, or covered by only overflowing queries. In the former case, the tuple of $D$ at $p$ could not have been retrieved, whereas in the latter, the algorithm could not rule out the possibility that $D$ had more than one tuple at $p$. In neither case could the algorithm have terminated.

Next we show that no resolved query $q$ covers more than one point in $S$. Otherwise, assume that $q$ contains $p_{1}$ and $p_{2}$ in $S$, in which case $q$ fully encloses the minimum bounding rectangle, denoted as $r$, of $p_{1}$ and $p_{2}$. Without loss of generality, suppose that $p_{1}\left(p_{j}\right)$ is from Group $i(j)$ such that $i \leq j$. If $i=j$, then $r$ contains the point $(i, \ldots, i)$, in which case at least $k+2$ tuples satisfy $q$ (i.e., $p_{1}, p_{2}$ and the $k$ diagonal tuples from Group $i$ ). Consider, on the other hand, $i<j$. In this scenario, the coordinate of $p_{1}$ is at most $i+1 \leq j$ on all attributes, while the coordinate of $p_{2}$ is at least $j$ on all attributes. Thus, $r$ contains the point $(j, \ldots, j)$, causing at least $k+2$ tuples to satisfy $q$ (i.e., $p_{1}, p_{2}$ and the $k$ diagonal tuples from Group $j$ ). Therefore, $q$ must overflow in any case, i.e., a contradiction.

The lemma indicates that at least $|S|=d m$ queries must be performed, which validates the correctness of Theorem 3 .

\subsection{Categorical Attributes}

First, if $d=1, U_{1}$ is a trivial lower bound on the cost of solving Problem 1. The reason is that, as long as $D$ has more than $k$ tuples, we must issue a query $A_{1}=c$ for every $c \in \operatorname{dom}\left(A_{1}\right)$ to verify whether a tuple exists at point $c$. For $d>1$, this subsection will establish:

THEOREM 4. Let $k, d, U$ be positive integers satisfying $d U^{2} \leq$ $2^{d / 4}, U \geq 3, k \geq 3$, and $d=2 k$. There is a dataset $D$ with $n=d U$ tuples in a d-dimensional categorical space, where each attribute has a domain size $U$, such that, any algorithm must use $\Omega\left(d U^{2}\right)$ queries to solve Problem 1 on $D$.

We thus know that our algorithm slice-cover (see Lemma 4) cannot be improved by more than a constant factor in the worst case, as shown below:

COROLlARY 2. In a categorical data space, no algorithm can guarantee solving Problem 1 with $o\left(\sum_{i=1}^{d} U_{i}+\right.$ $\left.\frac{n}{k} \sum_{i=1}^{d} \min \left\{U_{i}, \frac{n}{k}\right\}\right)$ queries. 


\begin{tabular}{|c|c|c|c|c|}
\hline & $A_{1}$ & $A_{2}$ & $\ldots$ & $A_{d}$ \\
\hline \multirow{3}{*}{ Group 0} & 1 & 0 & $\ldots$ & 0 \\
\hline & 0 & 1 & $\ldots$ & 0 \\
\hline & $\vdots$ & $\vdots$ & $\vdots$ & $\vdots$ \\
\hline \multirow{5}{*}{ Group 1} & 0 & 0 & $\ldots$ & 1 \\
\hline & 2 & 1 & $\ldots$ & 1 \\
\hline & 1 & 2 & $\ldots$ & 1 \\
\hline & $\vdots$ & $\vdots$ & : & $\vdots$ \\
\hline & 1 & 1 & $\ldots$ & 2 \\
\hline \multirow{4}{*}{ Group $U-1$} & 0 & $U-1$ & $\ldots$ & $U-1$ \\
\hline & $U-1$ & 0 & $\ldots$ & $U-1$ \\
\hline & $\vdots$ & $\vdots$ & : & $\vdots$ \\
\hline & $U-1$ & $U-1$ & $\ldots$ & 0 \\
\hline
\end{tabular}

Figure 8: A hard categorical dataset

Proof. In the setting of Theorem $4, \frac{n}{k}=\frac{d U}{k}=2 U$. Furthermore, $U_{1}=\ldots=U_{d}=U$. Hence, $\frac{n}{k} \sum_{i=1}^{d} \min \left\{U_{i}, \frac{n}{k}\right\}=$ $\frac{d U}{k} \cdot d U=2 d U^{2}$. Thus, the complexity in the corollary is $o\left(d U+d U^{2}\right)=o\left(d U^{2}\right)$. Hence, if the corollary was wrong, there would be an algorithm solving Problem 1 on the inputs in Theorem 4 with $o\left(d U^{2}\right)$ queries, which is a contradiction.

The rest of the subsection serves as the proof of Theorem 3. Our discussion is based on a hard dataset $D$ illustrated in Figure 8. Formally, $D$ consists of $U$ groups, each of which has $d$ tuples. In the $i$-th $(0 \leq i \leq U-1)$ group, for each attribute $A_{j}(1 \leq j \leq d)$, there is a tuple that takes value $(i+1) \bmod U$ on $A_{j}$, and value $i$ on all the other $d-1$ attributes. In other words, the data space $\mathbb{D}$ is $[0, U-1]^{d}$, although readers should be reminded that using integers to represent the values of a (categorical) attribute is purely for convenience, and that the ordering of those integers is irrelevant.

As before, we say that a query covers a point $p \in \mathbb{D}$ if $p$ satisfies the query. The next lemma gives an important fact:

LEMMA 6. If an algorithm has solved Problem 1 on our constructed $D$, then every point in $\mathbb{D}$ must be covered by at least one resolved query already performed.

Proof. We say that a point in $\mathbb{D}$ is empty if $D$ has no tuple at that point. Assume the existence of a point $p \in \mathbb{D}$ that is not covered in any resolved query. Hence, $p$ is either outside all the queries the algorithm issued, or is covered only by the overflowing queries. Hence, if $p$ was empty, the algorithm got no hint as to whether a tuple exists at $p$, and therefore, could not have terminated. On the other hand, if $p$ was not empty, the algorithm saw a tuple at $p$ but could not decide whether $D$ had any other tuple at $p$ (i.e., duplicates). In this case, the algorithm could not have terminated either. Thus we have a contradiction.

We will show that any correct algorithm must perform $\Omega\left(d U^{2}\right)$ resolved queries. Recall that, on each attribute $A_{i}(1 \leq i \leq d)$, a query $q$ has either a wildcard predicate $A_{i}=\star$, or a constant predicate $A_{i}=c$ for some $c \in[0, U-1]$. We say that $q$ is diverse, if it has at least two non-wildcard predicates with different constants specified. For example, the query with predicates $A_{1}=$ $1, A_{2}=2, A_{3}=\star, \ldots, A_{d}=\star$ is diverse, and so is the query with $A_{1}=1, A_{2}=1, A_{3}=2, A_{4}=\star, \ldots, A_{d}=\star$ (due to the predicates on $A_{2}$ and $A_{3}$ ), whereas the query with $A_{1}=1, A_{2}=$ $1, A_{3}=\star, \ldots, A_{d}=\star$ is not (as the same constant appears in the non-wildcard predicates).

LEMMA 7. A diverse query $q$ has at most two qualifying tuples, and hence, is always resolved (since $k>2$ ).
PROOF. Let $c_{1} \neq c_{2}$ be constants, each of which appears in a non-wildcard predicate of $q$. Suppose $c_{1}<c_{2}$. Two tuples from different groups cannot satisfy $q$ simultaneously. Otherwise, assume that tuple $t_{1}$ from group $i$ and tuple $t_{2}$ from group $j$ qualify $q$, and that $i<j$ without loss of generality. As $t_{1}$ contains only $i$ and $i+1$ in its attributes, we know $c_{1}=i$ and $c_{2}=i+1$. If $j<U-1, t_{2}$ contains only $j$ and $j+1$ in its attributes. We thus require $c_{1}=j=i$, which violates $i<j$. If $j=U-1, t_{2}$ contains only 0 and $U-1$ in its attributes. In this case, $c_{1}=0=i$ and $c_{2}=U-1=i+1$, which is also impossible because $U>2$.

On the other hand, it is easy to see that, no three tuples from the same group can together take value $c_{1}$ on one attribute, and also, value $c_{2}$ on another attribute. The lemma then follows.

We say that a query $q$ is monotonic, if (i) $q$ has at least two nonwildcard predicates, and (ii) the same constant is specified in all the non-wildcard predicates. For example, the query with predicates $A_{1}=1, A_{2}=1, A_{3}=\star, \ldots, A_{d}=\star$ is monotonic, whereas the query with $A_{1}=1, A_{2}=2, A_{3}=\star, \ldots, A_{d}=\star$ is not (as different constants are used in the non-wildcard predicates), and neither is the query with $A_{1}=1, A_{2}=\star, \ldots, A_{d}=\star$ (as it has only one non-wildcard predicate).

LEMMA 8. A resolved monotonic query $q$ has at least $d / 2$ nonwildcard predicates, and hence, covers at most $2^{d / 2}$ points in $\mathbb{D}$.

PROOF. Let $c$ be the constant in all the non-wildcard predicates of $q$. If $q$ has $\lambda \geq 2$ non-wildcard predicates, it retrieves exactly $d-\lambda$ tuples from group $c$, and no tuple from any other group. Hence, for $q$ to be resolved, $d-\lambda$ cannot exceed $k$, that is, $\lambda \geq$ $d-k=d / 2$.

It turns out that if a query is resolved, it must be either diverse or monotonic. In fact, if a query $q$ is neither diverse nor monotonic, it has at most one non-wildcard predicate. Such $q$ must retrieve at least $d$ tuples and hence, overflow (recall that $d=2 k>k$ ).

Given two different integers $x, y$ in $[0, U-1]$, we define a bichromatic set $S(x, y)$ of points in $\mathbb{D}$ :

for each $i \in[1, d], S(x, y)$ includes all the points that take $x$ or $y$ as their values on attribute $A_{i}$, except points $(x, x, \ldots, x)$ and $(y, y, \ldots, y)$.

For example, for $d=3$ and $U=3, S(1,2)=\{(1,1,2),(1,2,1)$, $(1,2,2),(2,1,1),(2,1,2),(2,2,1)\}$. That is, $S(1,2)$ has all the points having only 1 or 2 as their coordinates, but does not contain $(1,1,1)$ and $(2,2,2)$. Clearly, there are $\left(\begin{array}{c}U \\ 2\end{array}\right)$ bichromatic sets, each of which has $2^{d}-2$ points.

We are ready to explain why $\Omega\left(d U^{2}\right)$ queries are necessary to settle Problem 1 on $D$. Our discussion considers only the situation where less than $\frac{d}{8}\left(\begin{array}{c}U \\ 2\end{array}\right)$ diverse queries are performed by the algorithm (otherwise, trivially there are $\frac{d}{8}\left(\begin{array}{c}U \\ 2\end{array}\right)=\Omega\left(d U^{2}\right)$ queries). By Lemma 6 , every point of each bichromatic set must be covered by some resolved query. If a query $q$ covers at least one point in a bichromatic set $S(x, y)$, we say that $q$ touches $S(x, y)$.

A diverse query can touch at most one bichromatic set. As there are less than $\frac{d}{8}\left(\begin{array}{c}U \\ 2\end{array}\right)$ diverse queries but $\left(\begin{array}{c}U \\ 2\end{array}\right)$ bichromatic sets, we can find a bichromatic set that is touched by less than $d / 8$ diverse queries. Let $S(\alpha, \beta)$ be that bichromatic set (for some $\alpha, \beta$ in $[0, U-1]$ ), and $Q$ the set of diverse queries touching it (thus, $|Q|<d / 8)$.

Consider any query $q \in Q$. Since $q$ touches $S(\alpha, \beta), q$ has two non-wildcard predicates $A_{i}=\alpha$ and $A_{j}=\beta$ for some $i, j \in[1, d]$ with $i \neq j$ (in case multiple pairs of $(i, j)$ satisfy this requirement, choose one arbitrarily). Refer to $A_{i}$ and $A_{j}$ as the salient attributes 
of $q$. It is clear that $q$ cannot cover those points $p \in S(\alpha, \beta)$ such that $p$ has value $\alpha$ on both salient attributes of $q$. Let salient $(Q)$ be the union of the salient attributes of all the queries in $Q$. Hence, $\mid$ salient $(Q) \mid<d / 4$. By the previous reasoning, if a point $p \in$ $S(\alpha, \beta)$ takes value $\alpha$ on all the attributes in $\operatorname{salient}(Q), p$ is not covered by any of the queries in $Q$. How many such $p$ are there? As $\mid$ salient $(Q) \mid<d / 4, p$ can still choose $\alpha$ or $\beta$ as its value on each of the at least $\frac{3}{4} d+1$ attributes outside salient $(Q)$. Excluding points $(\alpha, \ldots, \alpha)$ and $(\beta, \ldots, \beta)$, we know that the number of such $p$ is at least $2^{1+3 d / 4}-2 \geq 2^{3 d / 4}$. As a remark, although we required $p$ to take $\alpha$ on the attributes in $\operatorname{salient}(Q)$, the argument works as well by taking $\beta$.

We have shown that at least $2^{3 d / 4}$ points in $S(\alpha, \beta)$ have not been covered by diverse queries. Those points must be covered by resolved monotonic queries, each of which, by Lemma 8, contains at most $2^{d / 2}$ points in $\mathbb{D}$. Therefore, the number of such queries must be at least $2^{3 d / 4} / 2^{d / 2}=2^{d / 4}$ which, as stated in Theorem 4 , is at least $d U^{2}$, thus completing the proof.

\section{EXTENSIONS: MIXED ATTRIBUTES}

This section will extend our techniques to handle a mixed data space $\mathbb{D}$ that has both numeric and categorical attributes. As defined in Section 1.1, we consider without loss of generality that the first cat attributes $A_{1}, \ldots, A_{\text {cat }}$ are categorical, and the other $d-c a t$ attributes $A_{c a t+1}, \ldots, A_{d}$ are numeric.

Define $\mathbb{D}_{\text {CAT }}$ as the categorical data (sub) space $\operatorname{dom}\left(A_{1}\right) \times$ $\ldots \times \operatorname{dom}\left(A_{\text {cat }}\right)$, namely, involving all and only the categorical attributes. Put differently, for any point $p \in D$, trimming off its coordinates on $A_{c a t+1}, \ldots, A_{d}$ gives a cat-dimensional point $p_{\mathrm{CAT}}$ in $\mathbb{D}_{\mathrm{CAT}}$. In a natural manner, $p_{\mathrm{CAT}}$ determines a set of points, which we denote by $\mathbb{D}_{\mathrm{NUM}}\left(p_{\mathrm{CAT}}\right)$, defined as:

$\mathbb{D}_{\text {NUM }}\left(p_{\text {CAT }}\right)$ is the set points $p^{\prime} \in \mathbb{D}$, such that $p^{\prime}$ shares the same value as $p_{\mathrm{CAT}}$ on every categorical attribute.

In fact, $\mathbb{D}_{\mathrm{NUM}}\left(p_{\mathrm{CAT}}\right)$ decides a $(d-c a t)$-dimensional numeric subspace of $\mathbb{D}$ : it includes all the numeric dimensions of $\mathbb{D}$, while fixing the categorical attributes to those of $p_{\text {CAT }}$.

As an example, the scenario of Figure 1 has four attributes, among which $c a t=2$ are categorical: $A_{1}=$ MAKE and $A_{2}=$ Body STYLE. Let $p_{\text {CAT }}=(\mathrm{BMW}$, sedan $)$. Then, $\mathbb{D}_{\mathrm{NUM}}\left(p_{\mathrm{CAT}}\right)$ includes all the points $p^{\prime} \in \mathbb{D}$ whose MAKE and BODY STYLE are BMW and sedan, respectively (but no constraint is imposed on the values of $p^{\prime}$ along the numeric attributes $A_{3}=$ PRICE and $A_{4}=$ Mileage).

Hybrid. Next, we present an algorithm, named hybrid, to solve Problem 1 in a mixed $\mathbb{D}$. The algorithm combines lazy-slice-cover (see Section 3.2) and rank-shrink (Section 2.3). Roughly speaking, it first enumerates all the points in $\mathbb{D}_{\mathrm{CAT}}$ using lazy-slice-cover and, when a point $p_{\mathrm{CAT}} \in \mathbb{D}_{\mathrm{CAT}}$ has been reached, invokes rank-shrink in the numeric subspace determined by $\mathbb{D}_{\mathrm{NUM}}\left(p_{\mathrm{CAT}}\right)$.

Now we provide the missing details. Recall that, lazy-slice-cover runs on a categorical server (i.e., one that supports only categorical attributes). To apply it on a server of our context here, we set a query's predicate on numeric attribute $A_{i}$ (for each $i \in[c a t+$ $1, d])$ to $A_{i} \in(-\infty, \infty)$. The effect is to disregard all the numeric attributes, and hence, essentially emulates a categorical server.

Also recall that lazy-slice-cover performs an (improved) depthfirst traversal on the data space tree $\mathbb{T}_{\mathrm{CAT}}$ built from $\mathbb{D}_{\mathrm{CAT}}$. Consider that it has come to a leaf node of $\mathbb{T}_{\mathrm{CAT}}$, or equivalently, a point $p_{\text {CAT }} \in \mathbb{D}_{\text {CAT }}$. In Section 3.2, the processing of $p$ finished with one extra query, but hybrid invokes rank-shrink upon $\mathbb{D}_{\mathrm{NUM}}\left(p_{\mathrm{CAT}}\right)$. Remember, however, that rank-shrink operates on a numeric server (i.e., one that supports only numeric attributes). To apply it on $\mathbb{D}_{\text {NUM }}\left(p_{\text {CAT }}\right)$, we fix a query's predicate on categorical attribute $A_{i}$ (for each $i \in[1, c a t]$ ) to $A_{i}=c_{i}$, where $c_{i}$ is the $A_{i}$ value of $p_{\text {CAT }}$. This effectively emulates a numeric server over the $(d-c a t)$ dimensional numeric subspace implied by $\mathbb{D}_{\mathrm{NUM}}\left(p_{\mathrm{CAT}}\right)$.

Upper bounds. Denote by $U_{i}(1 \leq i \leq c a t)$ the domain size of the $i$-th categorical attribute $A_{i}$. The following lemma gives the performance guarantees of hybrid, and establishes the last two bullets of Theorem 1 .

LEMMA 9. When cat $>1$, hybrid performs $\frac{n}{k} \sum_{i=1}^{c a t} \min \left\{U_{i}\right.$, $\left.\frac{n}{k}\right\}+\sum_{i=1}^{c a t} U_{i}+O((d-c a t) n / k)$ queries. When cat $=1$, the number of queries is $U_{1}+O(d n / k)$.

PROOF. We focus on only cat $>1$ because the same argument also applies to $c a t=1$. For $c a t>1$, the term $\frac{n}{k} \sum_{i=1}^{c a t} \min \left\{U_{i}\right.$, $\left.\frac{n}{k}\right\}+\sum_{i=1}^{c a t} U_{i}$ is the cost of running lazy-slice-cover, and follows directly from Lemma 4.

Let $S$ be all the leaf nodes of $\mathbb{T}_{\mathrm{CAT}}$ accessed by hybrid, namely, an instance of rank-shrink was executed on each node $u \in S$. Let $n_{u}$ be the number of tuples in $D$ having the same value as $u$ on every categorical attribute - these are the tuples in $\mathbb{D}_{\mathrm{NUM}}\left(p_{\mathrm{CAT}}\right)$ where $p_{\mathrm{CAT}}$ is the point in $\mathbb{D}_{\text {CAT }}$ corresponding to $u$. As disjoint bags of tuples are counted by the $n_{u}$ of different $u$, we have $\sum_{u \in S} n_{u} \leq n$. By Lemma 2, the instance of rank-shrink at $u$ issues $O\left((d-\right.$ cat $\left.) n_{u} / k\right)$ queries. Hence, the total number of queries issued by rank-shrink is $O\left((d-c a t) \sum_{u \in S}\left(n_{u} / k\right)\right)=O((d-c a t) n / k)$.

Lower bounds. We conclude this section by explaining why it is not possible to improve the upper bounds in Lemma 9 by more than a constant factor in the worst case. In fact, since a mixed data space is more general than both categorical and numeric spaces, the lower bounds in Section 4.1 and 4.2 are still applicable here. For example, if $c a t=0$, a mixed $\mathbb{D}$ becomes numeric. Hence, if there was an algorithm guaranteed faster than hybrid by nonconstant times, that algorithm would terminate with $o(d m)$ queries on the inputs stated in Theorem 3, giving a contradiction. On the other hand, if $c a t=d$, a mixed $\mathbb{D}$ becomes categorical. Similarly, if there was an algorithm faster than hybrid by non-constant times, that algorithm would terminate with $o\left(d U^{2}\right)$ queries on the inputs in Theorem 4, again giving a contradiction. The above discussion has taken care of $c a t>1$ or $c a t=0$, but an analogous argument apparently applies to the remaining case $c a t=1$ as well. This, together wish Theorems 3 and 4, establish Theorem 2.

\section{EXPERIMENTS}

In this section, we empirically evaluate the proposed techniques, and establish their superiority over alternative solutions.

Data. Our experiments were based on three real datasets, whose attributes, as well as the domain size of each attribute, are given in Figure 9 (where num indicates a numeric attribute). Specifically:

- Yahoo contains 69,768 tuples in a hidden database at autos.yahoo.com. Each tuple depicts 6 attributes of a vehicle. This is a mixed dataset (due to the presence of both numeric and categorical attributes).

- NSF contains 47,816 tuples in a hidden database at nsf.gov/awardsearch. Each tuple has 9 attributes of an NSF award. This is a categorical dataset.

- Adult contains 45,222 tuples in a census dataset that can be downloaded from archive.ics.uci.edu/ml/datasets/adult. Each tuple describes 14 attributes of a person working in the US. As with Yahoo, this is also a mixed dataset. 


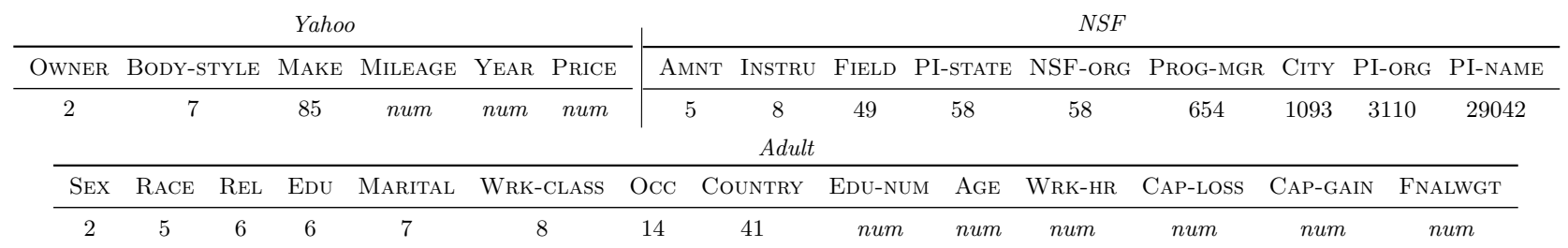

Figure 9: Attributes and their domain sizes of the datasets deployed

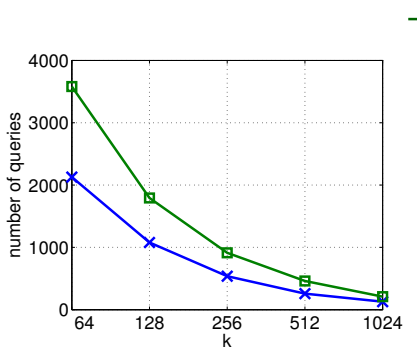

(a) Cost vs. $k(d=6)$

$\square$-binary-shrink — rank-shrink

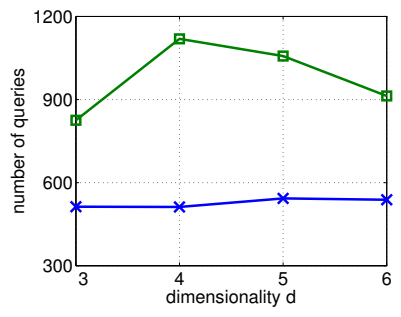

(b) Cost vs. $d(k=256)$

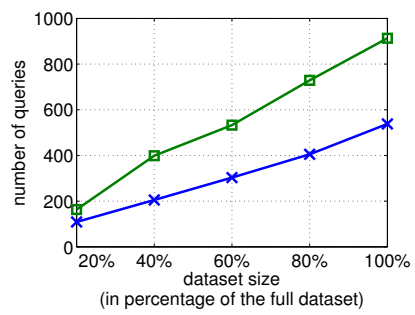

(c) Cost vs. $n(k=256, d=6)$

Figure 10: Query cost of numeric algorithms (dataset Adult-numeric)

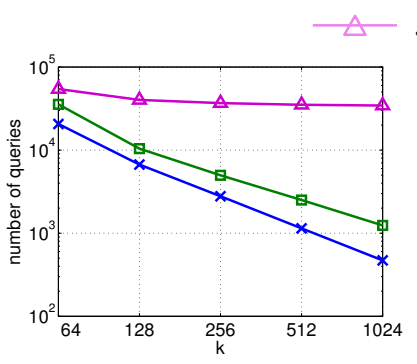

(a) Cost vs. $k(d=6)$

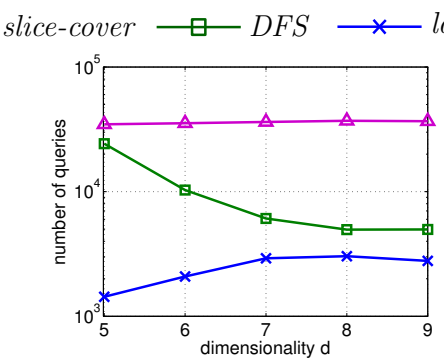

(b) Cost vs. $d(k=256)$ lazy-slice-cover

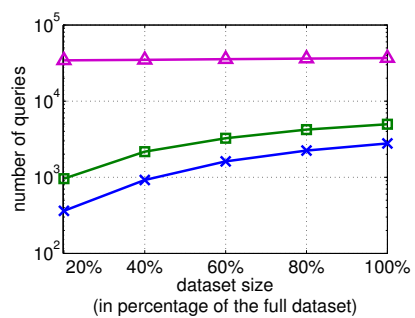

(c) Cost vs. $n(k=256, d=9)$

Figure 11: Query cost of categorical algorithms (dataset NSF)

We also extracted a numeric dataset from Adult, by including only its numeric attributes. The resulting dataset, named Adult-numeric, therefore has the same cardinality and dimensionality as Adult.

Recall that every algorithm in Sections 2, 3 and 5 works with an ordering of the attributes in the underlying dataset (i.e., which attribute is $A_{1}$, which one is $A_{2}$, and so on). The ordering is exactly as shown in Figure 9 (from left to right in each table), and is the same for all algorithms. Since our experiments require adjusting the value of $k$, we implemented a local server to run our algorithms. Our implementation conforms strictly to the problem setup in Section 1.1, so that the cost reported would be equivalent if the algorithms were executed on a remote web server. In a dataset, each tuple is assigned a random priority, so that if a query overflows, always the $k$ tuples with the highest priorities are returned.

Numeric algorithms. We start by studying the performance of numeric algorithms binary-shrink and rank-shrink in Section 2, using dataset Adult-numeric. For each algorithm, Figure 10a shows the number of queries it issued to extract Adult-numeric as a function of $k$. Setting $k$ to the median value 256, Figure $10 \mathrm{~b}$ plots their efficiency as the dimensionality $d$ varies from 3 to 6 . In this experiment, for each $d \in[3,6]$, we created a $d$-dimensional dataset by taking the $d$ attributes of Adult-numeric that have the highest numbers of distinct values. Specifically, the attribute with the most distinct values is FNALWGT, the second is CAP-GAIN, followed by CAP-LOSS, WrK-HR, AGE and EdU-NUM. Using $k=256$ and fixing $d$ to its original value 6 , Figure $10 \mathrm{c}$ compares the two algorithms with respect to the dataset size $n$. Here, a 20\% dataset corre- sponds to a random sample set of Adult-numeric, by independently sampling each of its tuples with a $20 \%$ probability. The datasets of the other percentages were generated in the same fashion.

Rank-shrink consistently outperformed binary-shrink in all cases. Furthermore, as predicted by our analysis in Section 2.3 (particularly, Lemma 2), the cost of rank-shrink was linear to $n$ and inversely linear to $k$ (to observe the inverse linearity, notice from Figure 10a that rank-shrink entailed half as many queries each time $k$ doubled). As a pleasant surprise, Figure $10 \mathrm{~b}$ demonstrates that the cost of rank-shrink stayed nearly the same as $d$ increased, even though Lemma 2 indicates that the cost should grow linearly in the worst case. In fact, by looking at the proof of Lemma 2, one would realize that the presence of $d$ in the final time complexity is due to 3-way splits (see Figure 2). Such a split happens only if many tuples share the same value on a certain attribute. As this is not true in Adult-numeric, 3-way splits were seldom performed, which explains the phenomenon in Figure 10b.

Categorical algorithms. We deployed a similar methodology to compare the efficiency of algorithms DFS, slice-cover and lazyslice-cover in Section 3. Note that DFS is in fact the baseline approach for crawling which was outlined in [15]. The underlying dataset was NSF. Figure 11 presents the results for the same set of experiments as in Figure 10. It is worth pointing out that, in Figure $11 \mathrm{~b}$, a $d$-dimensional dataset $(d \in[5,9])$ was generated from $N S F$ in the way as mentioned earlier for Figure 10b the number of distinct values on each attribute equals the attribute's domain size, which can be found in Figure 9). 


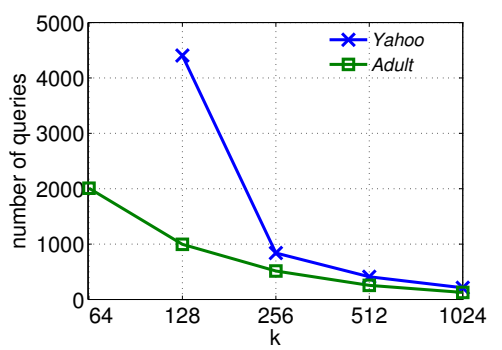

Figure 12: Cost of the mixed algorithm hybrid

Interestingly, slice-cover, even being asymptotically optimal, turned out to exhibit the worst performance. Of course, this does not contradict our theoretical analysis, because the optimality of slice-cover is reflected on the hardest dataset (see, for example, Figure 8). NSF is not such a dataset; hence, slice-cover does not guarantee better efficiency than a suboptimal solution like DFS. It is important to note that lazy-slice-cover was the clear winner in all the experiments (notice that the y-axes of all diagrams in Figure 11 are in log scale). The huge improvement of lazy-slice-cover over slice-cover confirms the necessity and effectiveness of the heuristic discussed in Section 3.2.

Hybrid algorithms. Having demonstrated the superiority of rankshrink and lazy-slice-cover over their competitors, next we evaluate the behavior of their combination: the hybrid algorithm in Section 5. For this purpose, we employed both mixed datasets Yahoo and Adult. Figure 12 illustrates the number of queries performed by hybrid to crawl each dataset entirely, as $k$ changes from 64 to 1024. Note that there is no reported value for Yahoo at $k=64$ because it has more than 64 identical tuples (i.e., they agree with each other on every dimension) - for the reason explained in Section 1.1, no algorithm can successfully extract the dataset in full when $k=64$.

In practice, it would be a nice property for a crawling algorithm to be able to return the tuples of a hidden database in a progressive manner. Namely, it should gradually churn out new tuples as it runs, instead of outputting most tuples only at the end. This property allows the crawler to terminate the algorithm at any moment, while still able to obtain a number of tuples proportional to the amount of time that has been spent. Motivated by this, the last set of experiments examines the progressiveness of hybrid. In Figure 13 (where $k$ was set to 256), for each dataset, we present the percentage of the tuples extracted (the y-axis) against the percentage of the queries issued (the $\mathrm{x}$-axis). For example, a point $(20 \%$, $30 \%$ ) in this figure means that, hybrid is able to discover $30 \%$ of the tuples in the dataset, at the moment when it has issued $20 \%$ of all the queries that eventually need to be performed. We were delighted to observe linear progressiveness for both datasets, as is clear from Figure 13.

\section{CONCLUSIONS}

Currently, search engines cannot effectively index hidden databases, and are thus unable to direct queries to the relevant data in those repositories. With the rapid growth in the amount of such hidden data, this problem has severely limited the scope of information accessible to ordinary Internet users. In this paper, we attacked an issue that lies at the heart of the problem, namely, how to crawl a hidden database in its entirety with the smallest cost. We have developed algorithms for solving the problem when the underlying dataset has only numeric attributes, only categorical attributes, or both. All our algorithms are asymptotically optimal, i.e., none of them can be improved by more than constant times in the worst

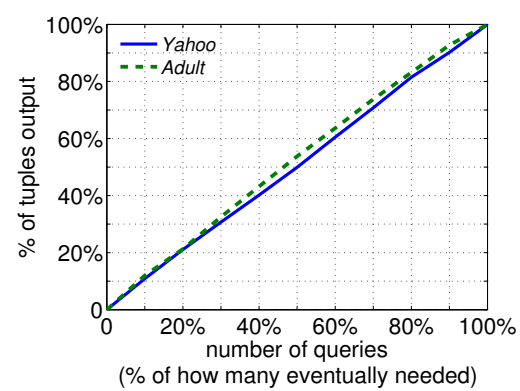

Figure 13: Output progressiveness of hybrid $(k=256)$

case. Our theoretical analysis has also revealed the factors that determine the hardness of the problem, as well as how much influence each of those factors has on the hardness.

\section{REFERENCES}

[1] E. Agichtein, P. G. Ipeirotis, and L. Gravano. Modeling query-based access to text databases. In WebDB, pages 87-92, 2003.

[2] M. Alvarez, J. Raposo, A. Pan, F. Cacheda, F. Bellas, and V. Carneiro. Crawling the content hidden behind web forms. In ICCSA, pages 322-333, 2007.

[3] Z. Bar-Yossef and M. Gurevich. Efficient search engine measurements. In $W W W$, pages 401-410, 2007.

[4] Z. Bar-Yossef and M. Gurevich. Mining search engine query logs via suggestion sampling. PVLDB, 1(1):54-65, 2008.

[5] L. Barbosa and J. Freire. Siphoning hidden-web data through keyword-based interfaces. JIDM, 1(1):133-144, 2010.

[6] N. Bruno, L. Gravano, and A. Marian. Evaluating top-k queries over web-accessible databases. In ICDE, pages 369-380, 2002.

[7] A. Cali and D. Martinenghi. Querying the deep web. In $E D B T$, pages 724-727, 2010.

[8] J. Callan and M. Connell. Query-based sampling of text databases. ACM TOIS, 19(2):97-130, 2001

[9] A. Dasgupta, X. Jin, B. Jewell, N. Zhang, and G. Das. Unbiased estimation of size and other aggregates over hidden web databases. In SIGMOD, pages 855-866, 2010

[10] E. Dragut, T. Kabisch, C. Yu, and U. Leser. A hierarchical approach to model web query interfaces for web source integration. $P V L D B$, 2(1):325-336, 2009.

[11] E. Dragut, C. Yu, and W. Meng. Meaningful labeling of integrated query interfaces. In $V L D B$, pages 679-690, 2006.

[12] B. He and K. Chang. Statistical schema matching across web query interfaces. In SIGMOD, pages 217-228, 2003.

[13] B. He, K. Chang, and J. Han. Discovering complex matchings across web query interfaces: A correlation mining approach. In $K D D$, pages 148-157, 2004.

[14] P. Ipeirotis and L. Gravano. Distributed search over the hidden web: Hierarchical database sampling and selection. In $V L D B$, pages 394-405, 2002.

[15] X. Jin, N. Zhang, and G. Das. Attribute domain discovery for hidden web databases. In SIGMOD, pages 553-564, 2011.

[16] S. Liddle, D. Embley, D. Scott, and S. Yau. Extracting data behind web forms. In ER (Workshops), pages 402-413, 2002.

[17] J. Madhavan, D. Ko, L. Kot, V. Ganapathy, A. Rasmussen, and A. Halevy. Google's deep-web crawl. PVLDB, 1(2):1241-1252, 2008.

[18] A. Ntoulas, P. Zerfos, and J. Cho. Downloading textual hidden web content through keyword queries. In $J C D L$, pages 100-109, 2005.

[19] S. Raghavan and H. Garcia-Molina. Crawling the hidden web. In $V L D B$, pages 129-138, 2001.

[20] K. Vieira, L. Barbosa, J. Freire, and A. Soares da Silva. Siphon++: a hidden-webcrawler for keyword-based interfaces. In CIKM, pages 1361-1362, 2008.

[21] Z. Zhang, B. He, and K. Chang. Understanding web query interfaces: best-effort parsing with hidden syntax. In SIGMOD, pages 107-118, 2004 